\title{
Is There a Role of Postoperative Radiation Therapy in Completely Resected Stage I/II Thymic Epithelial Tumor?
}

\author{
Dongryul Oh, $\mathrm{MD}^{1}$ \\ Yong Chan Ahn, MD, PhD' \\ Kwhanmien Kim, MD, PhD2 \\ Jhingook Kim, MD, PhD' \\ Young Mog Shim, MD, PhD² \\ Jungho Han, MD, $\mathrm{PhD}^{3}$
}

Departments of ${ }^{\prime}$ Radiation Oncology,

${ }^{2}$ Thoracic Surgery, and ${ }^{3}$ Pathology,

Samsung Medical Center,

Sungkyunkwan University School

of Medicine, Seoul, Korea

\begin{abstract}
Purpose
Retrospective analyses of patients with stage I-II thymic epithelial tumors (TET) who were treated with either surgery alone (S) or surgery plus postoperative radiation therapy (SRT) were conducted to evaluate the role of adjuvant radiation therapy (RT).
\end{abstract}

\section{Materials and Methods}

A total of 110 stage I-II TET patients following complete resection were included in this study. Postoperative radiation therapy was recommended for those with aggressive histologic type and/or invasive features according to the surgeons' judgment during the operation. A median dose of $54.0 \mathrm{~Gy}$ (range, 44 to $60 \mathrm{~Gy}$ ) focused on the primary tumor bed was administered to 57 patients $(51.8 \%)$.

\section{Results}

In all patients, the rates of overall survival, disease-specific survival, and disease-free survival at 10 years were $91.7 \%, 97.1 \%$, and $95.8 \%$, respectively. No significant differences in disease-specific survival ( $100 \%$ in the $S$ group and $93.5 \%$ in the SRT group at 10 years, $p=0.12$ ) and disease-free survival (98.1\% in the $S$ group and $94.5 \%$ in the SRT group at 10 years, $p=0.41$ ) were observed between the treatment groups, although a significantly larger number of World Health Organization (WHO)-type B2-C $(p<0.001)$ and Masaoka stage II $(p=0.03)$ tumors were observed in the SRT group than in the $S$ group. No local recurrence was observed in the SRT group. No grade 2 or greater RT-related toxicities were observed in the SRT group.

\section{Conclusion}

Excellent outcomes were achieved in patients with stage I-II TET who underwent complete resection. Considering excellent local control and low morbidity, adjuvant RT may be considered in high risk patients with WHO-type B2-C histology and Masaoka stage II.

Department of Radiation Oncology,

Samsung Medical Center,

Sungkyunkwan University School of Medicine,

81 Irwon-ro, Gangnam-gu, Seoul 135-710, Korea

Tel: $82-2-3410-2602$

Fax: 82-2-3410-2619

E-mail: ahnyc@skku.edu

Received June 11, 2012

Accepted August 21, 2012

\section{Key words}

Thymic neoplasms, Surgery, Adjuvant radiotherapy

\section{Introduction}

Thymic epithelial tumor (TET) is an indolent tumor originating from epithelial cells within the thymic gland. It is often associated with autoimmune disorders, such as myasthenia gravis and red cell aplasia [1]. In 1999, the World Health Organization (WHO) adopted a pathologic classification system based on the morphology of epithelial cells as well as the lymphocyte-to-epithelial cell ratio [2]. They classified TET into type $\mathrm{A}, \mathrm{AB}, \mathrm{B} 1, \mathrm{~B} 2$, and B3, and the more heterogeneous group of thymic carcinoma, type C. When compared to WHO-type A-B1 tumors, WHOtype B2-C tumors are more likely to have an aggressive clinical course [3-6]. The widely used staging system, which was developed by Masaoka et al. in 1981 [7], and modified in 1994 [8,9], describes TET in terms of the local extension of the tumor and classified it according to four stages: completely encapsulated tumor, stage I; transcapsular invasion or surrounding fatty tissue invasion, stage II; neighboring organ invasion, stage III; and metastasis to pleura, pericardium, lymph node, or distant site, stage IV.

Complete surgical resection is the mainstay treatment for patients with 
TET. Adjuvant radiation therapy (RT) is usually advocated for patients with incompletely resected tumors or Masaoka stage III. Conversely, the issue of adjuvant RT in completely resected early stage TET has been controversial, especially in stage II [10-14]. At the author's institute, adjuvant RT has been recommended for patients with early stage TET with aggressive WHO histology and/or invasiveness according to the surgeon's judgment during the operation; retrospective analyses were performed in order to evaluate the role of adjuvant RT.

\section{Materials and Methods}

\section{Patients}

Between January 1995 and December 2004, 201 patients were registered as having TET and underwent surgical resection at the authors' institute. All of the records of these patients were reviewed and the stages were classified according to the Masaoka staging system. A total of 117 patients were Masaoka stage I/II, and, after excluding seven ineligible patients, retrospective analyses were performed on 110 patients. Reasons for exclusion from the current analyses included microscopic residual tumor in four patients with Masaoka stage II, unavailable follow-up information in two, and synchronous double primary cancer in one, respectively.
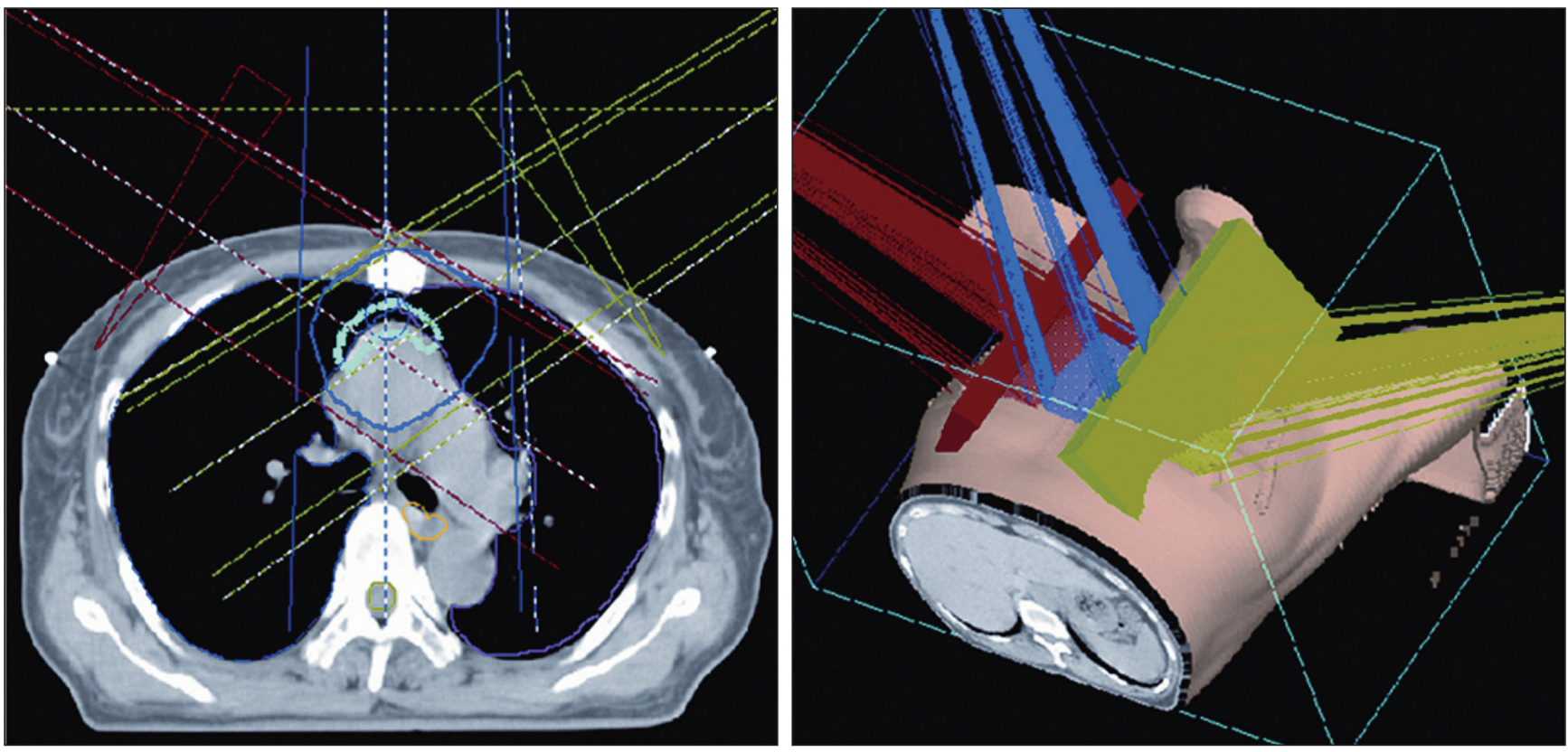

Fig. 1. The typical beam arrangement of three-dimensional conformal radiation therapy (anterior/right anterior oblique/left anterior oblique).

\section{Pathologic review}

Review of all specimens and classification of histologic subtypes were performed by the pathologist in our institution $(\mathrm{JHH})$. All cases before 1999, which were initially categorized according to Muller-Hermelink classification, were reclassified according to the WHO classification. To distinguish TET from other mediastinal tumors, immunohistochemical staining was performed using $1: 50 \mathrm{CD} 5,1: 100$ cytokeratin, $1: 50$ MIC-2 (CD99), $1: 100$ leucocyte common antigen, and $1: 40$ placental leucocyte alkaline phosphatase.

\section{Treatment}

Total thymectomy was performed via median sternotomy in 80 patients (72.7\%), by video-assisted thoracoscopy in 25 patients $(22.7 \%)$, by thoracotomy in four patients (3.6\%), and by trans-cervical incision in one patient $(0.9 \%)$, respectively. All patients underwent complete resection, defined as no macroscopic or microscopic residual tumor. Adjuvant RT was recommended for patients with either a WHO-type B2-C tumor (including non-medullary type according to Muller-Hermelink classification before 1999) or Masaoka stage II. In addition, the surgeon's judgment regarding tumor invasiveness or adhesion observed during the operation was also considered.

Adjuvant RT was started at four to six weeks after surgery. The radiation target volume was delineated on the computed tomography (CT) scan taken for simulation, which included the tumor bed. All relevant clinical information, including preoperative images, record of surgery, pathologic report, and surgical clips seen on simulation CT were referenced in the 
target delineation. The typical beam arrangement was an anterior 3-field wedge technique (anterior/right anterior oblique/left anterior oblique) (Fig. 1). Using 6-10 MV X-ray, a total median dose of 54 Gy (range, 44 to $60 \mathrm{~Gy}$ ) was delivered over a period of five to six weeks by daily 1.8 Gy or $2.0 \mathrm{~Gy}$. In the current study, none of the patients received adjuvant chemotherapy.

\section{Statistical analysis}

The Kaplan-Meier method was used for determination of survival rate. Survival duration was calculated from the dates of surgery to the dates of the last follow-up or any events such as death or recurrence. The log-rank test was used for comparison of differences between the groups, and the Cox proportional hazards regression model was used for multivariate analysis. The Chi-square test was used for analysis of the distribution of categorical variables, and the t-test was used for analysis of the distribution of continuous variables. A p-value less than 0.05 was considered statistically significant and the software SPSS ver. 19.0 (SPSS Inc., Chicago, IL) was used for analysis.

\section{Results}

\section{Patients' characteristics}

All patients underwent complete resection of tumor. Among 110 patients, 53 patients (48.2\%) underwent surgery alone group (S group), while 57 (51.8\%) received surgery plus postoperative RT group (SRT group). Eighty patients (72.7\%) had Masaoka stage I and 30 patients (27.3\%) had Masaoka stage II. The clinical characteristics of all patients are shown in Table 1. SRT group had a significantly large number of patients with Masaoka stage II compared to $S$ group $(\mathrm{p}=0.03)$. The WHO types were as follows: type $\mathrm{A}$ in six (5.5\%); type $\mathrm{AB}$ in 35 (31.8\%); type $\mathrm{B} 1$ in $19(17.3 \%)$; type $\mathrm{B} 2$ in $30(27.3 \%)$; type B3 in $15(13.6 \%)$; and type $\mathrm{C}$ in five (4.5\%). The proportion of WHO-type B2-C tumors was much higher in the SRT group than in the S group $(18.9 \%$ and $70.2 \%$, $\mathrm{p}<0.001$ ). A summary of the distribution of WHO types according to Masaoka stage and treatment group is shown in Table 2. The median follow-up duration for all patients was 110 months (range, 4 to 197 months) and 114 months (range, 60 to 197 months) for living patients.

Table 1. Clinical characteristics of 110 patients with early stage thymoma

\begin{tabular}{|c|c|c|c|}
\hline Characteristics & Surgery alone $(\mathrm{n}=53)$ & Surgery plus radiotherapy $(\mathrm{n}=57$ ) & p-value \\
\hline Mean age (SD, yr) & $48.56(13.96)$ & $49.61(11.29)$ & 0.66 \\
\hline Gender & & & 1.00 \\
\hline Male & 28 & 30 & \\
\hline Female & 25 & 27 & \\
\hline Mysathenic symptom & & & 1.00 \\
\hline Yes & 12 & 12 & \\
\hline No & 41 & 46 & \\
\hline Mean tumor size (SD, cm) & $5.66(2.69)$ & $6.85(3.17)$ & 0.04 \\
\hline Masaoka stage & & & 0.03 \\
\hline I & 44 & 36 & \\
\hline II & 9 & 21 & \\
\hline WHO-type & & & $<0.001$ \\
\hline A & 5 & 1 & \\
\hline $\mathrm{AB}$ & 25 & 10 & \\
\hline $\mathrm{B} 1$ & 13 & 6 & \\
\hline B2 & 9 & 21 & \\
\hline B3 & 1 & 14 & \\
\hline $\mathrm{C}$ & 0 & 5 & \\
\hline WHO-type group & & & $<0.001$ \\
\hline A-B1 & 43 & 17 & \\
\hline B2-C & 10 & 40 & \\
\hline Operative procedure & & & 0.20 \\
\hline Median sternotomy & 34 & 46 & \\
\hline Thoracotomy & 3 & 1 & \\
\hline Transcervical approach & 1 & 0 & \\
\hline Video-assisted thoracoscopy & 15 & 10 & \\
\hline
\end{tabular}

SD, standare deviation; WHO, World Health Organization. 
Table 2. Distribution of WHO histologic type according to Masaoka stage and treatment group

\begin{tabular}{|c|c|c|c|c|}
\hline \multirow{2}{*}{ WHO-type } & \multicolumn{2}{|c|}{ Masaoka stage I } & \multicolumn{2}{|c|}{ Masaoka stage II } \\
\hline & Surgery alone & Surgery plus radiotherapy & Surgery alone & Surgery plus radiotherapy \\
\hline A-B1 & 36 & $9^{\text {a) }}$ & 7 & 8 \\
\hline $\mathrm{B} 2-\mathrm{C}$ & $8^{\text {b) }}$ & 27 & 2 & 13 \\
\hline Total & 44 & 36 & 9 & 21 \\
\hline
\end{tabular}

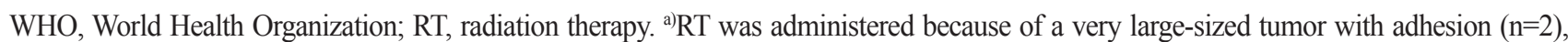
non-medullary type according to Muller-Hermelink classification before $1999(\mathrm{n}=5)$, surgeon's judgment for local invasiveness during the operation $(n=2),{ }^{b}$ RT was not administered due to patients' refusal $(n=2)$, surgeon's judgment for local invasiveness during the operation $(n=6)$.

Table 3. Characteristics of four patients who experienced recurrence during the follow-up period

\begin{tabular}{|c|c|c|c|c|c|c|c|}
\hline No. & $\begin{array}{c}\text { Gender/Age } \\
\text { (yr) }\end{array}$ & $\begin{array}{l}\text { Masaoka } \\
\text { stage }\end{array}$ & WHO-type & $\begin{array}{l}\text { Initial operation } \\
\text { procedure }\end{array}$ & $\begin{array}{l}\text { Adjuvant } \\
\text { RT }\end{array}$ & $\begin{array}{l}\text { Failure site } \\
\text { (time to } \\
\text { recurrence, mo) }\end{array}$ & $\begin{array}{lc}\text { Salvage } & \text { Final status } \\
\text { therapy } & \text { (time after salvage therapy or } \\
& \text { after recurrence, } \text { mo) }\end{array}$ \\
\hline 1 & $\mathrm{M} / 29$ & 1 & B2 & Median sternotomy & No & Local (24) & Alive with NED (113) \\
\hline 2 & $\mathrm{M} / 53$ & 1 & B2 & $\begin{array}{l}\text { Video-assisted } \\
\text { thoracoscopy }\end{array}$ & Yes & Pleural seeding (43) & Alive with NED (43) \\
\hline 3 & $\mathrm{M} / 53$ & 1 & B2 & $\begin{array}{l}\text { Video-assisted } \\
\text { thoracoscopy }\end{array}$ & Yes & Pleural seeding (55) & Dead of disease (27) \\
\hline 4 & $\mathrm{M} / 58$ & 2 & $\mathrm{C}$ & Median sternotomy & Yes & Liver, bone (18) & $\begin{array}{l}\text { Palliative } \\
\text { chemotherapy }\end{array}$ Dead of disease (90) \\
\hline
\end{tabular}

WHO, World Health Organization; RT, radiation therapy; M, male; NED, no evidence of diesease.

Table 4. Intercurrent deaths by stages and treatment groups

\begin{tabular}{lllc} 
& Stage I & Stage II & Total \\
Surgery & 1 (hepatocellular carcinoma) & 1 (aplastic anemia) & 2 \\
Surgery plus RT & 4 (old age, pneumonia, myasthenia) & 1 (unknown ) & 5 \\
Total & 5 & 2 & 7 \\
\hline
\end{tabular}

RT, radiation therapy.

\section{Failure patterns and causes of deaths}

Four patients had recurrence during the follow-up period: local recurrence in one patient; pleural seeding in two; and distant metastases (liver and bone) in one (Table 3). Of these four with recurrence, two patients have remained alive until the current analyses. One patient with WHO-type B2 and Masasoka stage I, who initially underwent surgery alone, developed local recurrence at the anterior mediastinum at 24 months after surgery. Treatment with high dose RT (50 Gy) resulted in successful salvage of this patient, who has remained disease-free for 113 months following the salvage treatment. The other patient with WHOtype B2 and Masaoka stage I, who initially underwent surgery and received adjuvant RT, developed pleural seeding at 43 months after surgery. Excision of the pleural mass and administration of adjuvant RT to the tumor bed were performed; he has also remained disease-free for 43 months following salvage treatment. The other two patients, one with pleural seeding and the other with distant metastasis, ultimately died as a result of disease progression.

In addition to two patients who died of TET recurrence and progression, seven patients died of intercurrent diseases other than TET (Table 4). In the $\mathrm{S}$ group, one patient with stage I died of hepatocellular carcinoma, and another patient with stage II died of aplastic anemia. In the SRT group, four patients with stage I and one with stage II died of intercurrent diseases: two due to old age; one pneumonia; one myasthenia; and one unknown.

\section{Clinical outcomes}

In all patients, the rates of overall survival (OS), disease-specific survival (DSS), and disease-free survival (DFS) at 10 years were $91.7 \%$, $97.1 \%$, and $95.8 \%$, respectively. No differences in OS (93.3\% and 86.2\%, $\mathrm{p}=0.60)$, DSS (98.4\% and 92.3\%, $\mathrm{p}=0.40)$, and DFS $(96.2 \%$ and $96.7 \%$, $\mathrm{p}=0.94$ ) were observed between Masaoka stages. Survival figures for the $\mathrm{S}$ group were better than those for the SRT group, without statistical 
Disease-specific survival

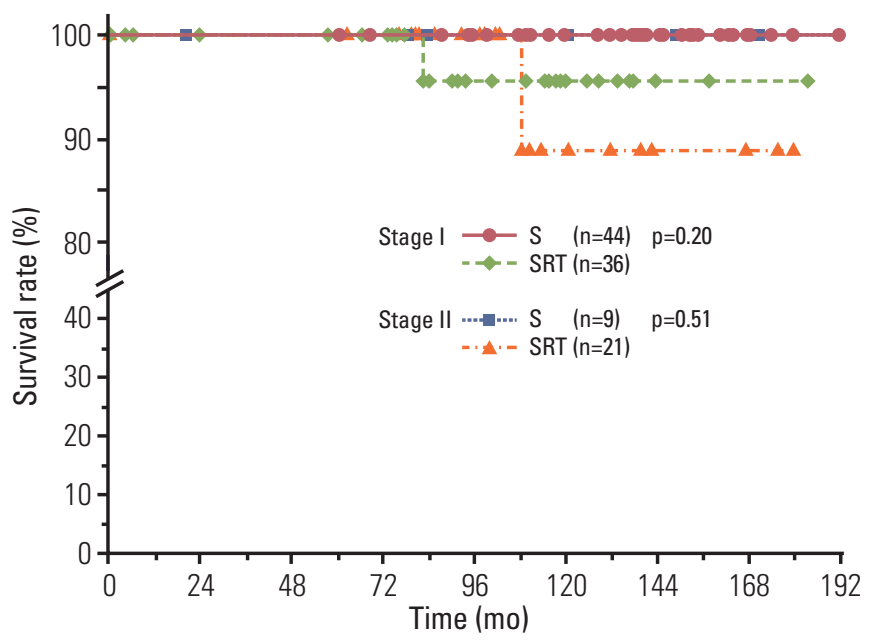

Disease-free survival

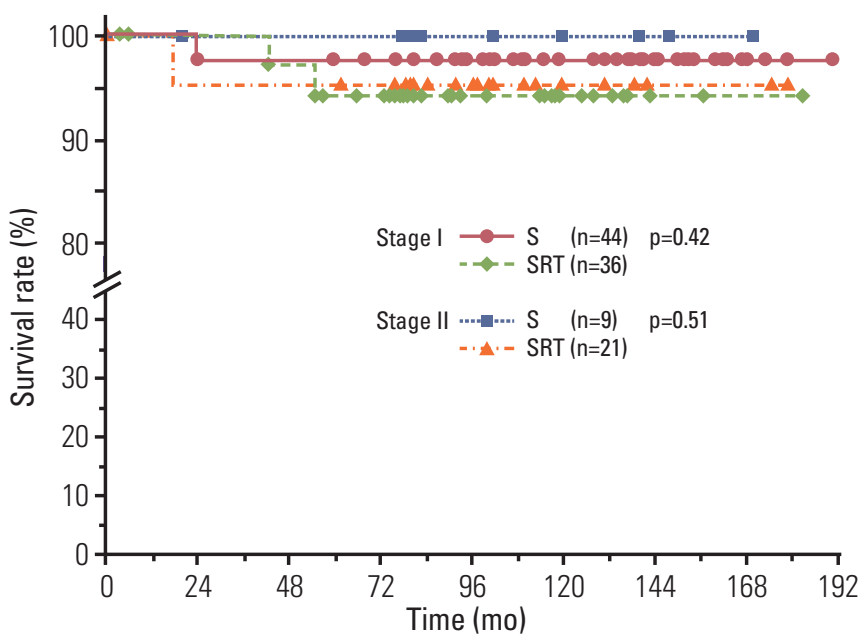

Fig. 2. Comparison of survival rates according to adjuvant radiation therapy (RT) in stage I and stage II thymic epithelial tumor. S, surgery alone; SRT, surgery plus RT.

ignificance: $98.1 \%$ and $85.2 \%$ in OS ( $\mathrm{p}=0.06) ; 100 \%$ and $93.5 \%$ in DSS $(\mathrm{p}=0.12)$; and $98.1 \%$ and $94.5 \%$ in DFS ( $\mathrm{p}=0.41)$. Patients with WHOtype A-B1 tumors had favorable outcomes at 10 years, compared to those with WHO-type B2-C: $98.3 \%$ and $83.5 \%$ in OS ( $\mathrm{p}=0.02) ; 100 \%$ and $93.0 \%$ in DSS $(\mathrm{p}=0.09)$; and $100 \%$ and $91.5 \%$ in DFS $(\mathrm{p}=0.02)$. Multivariate analyses were performed for OS, DSS, and DFS, including the variables of WHO-type, Masaoka stage, presence of myasthenia gravis symptoms, administration of RT, and tumor size; no significant factor was found.

\section{Subgroup analysis according to Masaoka stage}

\section{1) Stage $I$}

Patients in the SRT group had worse 10-year OS than those in the S group $(100 \%$ and $84.5 \%, \mathrm{p}=0.02)$, however, no significant differences were observed in DSS (100\% and 95.7\%, $\mathrm{p}=0.20)$ and DFS $(97.7 \%$ and $94.1 \%, \mathrm{p}=0.42$ ) at 10 years, respectively (Fig. 2). Compared to patients with WHO-type B2-C, those with WHO-type A-B1 had significantly better DFS $(p=0.04)$, but not OS $(p=0.12)$ or DSS $(p=0.20)$.

\section{2) Stage II}

No significant differences in 10 -year OS ( $88.9 \%$ and $84.7 \%, \mathrm{p}=0.90)$, DSS ( $100 \%$ and $88.9 \%, \mathrm{p}=0.51)$, and DFS ( $100 \%$ and $95.2 \%, \mathrm{p}=0.51)$ were observed between the treatment groups (Fig. 2). Patients with WHOtype A-B1 showed a tendency for a better prognosis in OS $(\mathrm{p}=0.07)$, but not in DSS $(\mathrm{p}=0.28)$ and DFS $(\mathrm{p}=0.32)$.

\section{3) Toxicity}

There was no postoperative mortality. Acute and late toxicity profiles following postoperative RT were evaluable in 51 patients in 57 in the SRT group (89.5\%). Grade I pneumonitis and esophagitis by Radiotherapy Oncology Group (RTOG) grading was observed in three (5.9\%) and four patients $(7.8 \%)$, respectively. No late cardiac and pulmonary toxicities were observed in the current study.

\section{Discussion}

Incidence of early stage TET is not high, and it usually shows a rather indolent clinical course. In general, follow-up for a long duration is needed in this setting in order to understand the clinical course and the effect of additional treatment modality to surgery. Overall, approximately $50 \%$ of deaths were attributable to TET and its treatment [11]. However, in the current study, among nine deaths, only two deaths were attributable to progression of TET after recurrence (22.2\%), while seven were intercurrent deaths unrelated to TET (77.8\%). Thus, DFS or DSS, rather than OS, were better indicators for evaluation of the effect of treatment. In the current study, both DFS and DSS did not differ between the treatment groups, although the SRT group had significantly more WHO-type B2-C $(p<0.001)$ and Masaoka stage II $(p=0.03)$ than the $S$ group. Recurrence rates after resection in Masaoka stage II are usually higher than those in stage I $[11,15]$ and WHO histology has been known as a prognosticator in TET [3,4].

Most authors have reported that complete surgical resection alone is sufficient for achievement of an excellent outcome in treatment of stage ITET. The five-year OS rates ranged from $89 \%$ to $100 \%$, and local control rate approached $100 \%$ [16]. In addition, results of one randomized trial demonstrated that the addition of RT following complete resection in stage ITET had no benefit [17]. Based on these data, adjuvant RT has not been 
usually recommended. However, some authors have advocated the use of adjuvant RT in selected patients with stage I TET. Regnard et al. [18] reported that the recurrence rate was significantly higher among patients with peritumoral adherence (five of 26 patients) when compared with those with no peritumoral adherence ( 0 of 109 patients) in stage I $(p=0.001)$. Ogawa et al. [19] also advocated the use of adjuvant RT in stage I patients if there had been peritumoral adherence, based on their observation of no failure among 10 patients with peritumoral adherence following postoperative RT. Among 80 patients with Masaoka stage I, the authors observed a total of three recurrences: one among subjects in the S group; and two among subjects in the SRT group. While two patients in the SRT group experienced pleural seeding, one patient in the S group experienced local recurrence, which could be prevented by adjuvant RT. Furthermore, the patient had WHO-type B2 histology. Of eight Masaoka stage I patients with WHO-type B2-C histology, one patient (12.5\%) experienced local recurrence. Although our results did not demonstrate definite evidence for the benefit of adjuvant RT in patients with Masaoka stage I, it implies that adjuvant RT, more or less, would be helpful with respect to local control, especially with WHO-type B2-C histology, but not to pleural or distant dissemination, as shown in other studies [10,19, 20], when considering that there was no local recurrence in the SRT group.

The use of adjuvant RT for stage II TET has remained controversial. The rationale of adjuvant RT in stage II TET was that it resulted in lowered recurrence rate, when compared to surgery alone. Curran et al. [21] reported on the recurrence rate after complete resection in patients with stage II TET. The local recurrence rate at five years was $47 \%$ in patients who did not receive adjuvant RT, and $0 \%$ in those who received RT. Monden et al. [22] also reported that, in stage II TET patients, the recurrence rate was $29 \%$ after complete resection alone, and $8 \%$ after postoperative RT. However, those reports could be criticized for being old data, with small sample sizes, and suboptimal outcomes following surgery alone. Patients treated with surgery plus RT showed generally excellent outcomes, with local control rates ranging from $90 \%$ to $100 \%[18,23,24]$. However, several authors have recently reported that no additional benefit was achieved by addition of RT after complete resection in patients with stage II TET. Rena et al. [12], in a study of 58 patients with stage II TET, reported that no difference in DFS ( $\mathrm{p}=0.432$ ) was observed regardless of adjuvant RT following complete resection. Korst et al. [25], through metaanalysis of 592 patients with stage II/III TET, reported no significant reduction in recurrence rate after adjuvant RT in stage II patients. Utsumi et al. [14] reviewed 324 patients who underwent complete resection of TET. In subgroup analysis, DSS of patients with stage II TET was $>90 \%$ regardless of the addition of adjuvant RT. Chen et al. [10], who conducted an analysis of 107 patients with stage II TET who underwent complete resection, reported no differences in 10-year DFS (93.1\% and 82.6\%, $\mathrm{p}=0.27$ ) and 10 -year DSS ( $97.5 \%$ and $89.3 \%, \mathrm{p}=0.97$ ) by addition of RT. Results of the current study also showed no differences in DFS and DSS between the treatment groups. However, based on these findings, the role of RT could hardly be speculated.

It should be noted that, in the current study, significantly different distribution in WHO histologic subtypes was observed between treatment groups: only two of nine patients (22.2\%) in the S group had WHO-type B2-C; whereas 13 of 21 (61.9\%) patients in the SRT group had WHOtype B2-C (Table 2). Histologic subtype of WHO-type B2-C has been well known as a poor prognosticator [3,4]. In addition, surgeons in the current study requested postoperative RT more frequently if they observed and perceived local invasiveness during surgery. In the current study, despite these obvious selection biases, no differences were observed in DFS and DSS.

In stage II TET, the recurrence rates after resection were usually reported as $11-14 \%$, which was usually higher than those of stage I $[11,15]$. Recent studies have reported the recurrence rate and the pattern of failures based on the histologic subtypes (Table 5). In WHO-type B2-C, more recurrences were usually encountered in patients who received surgery plus RT, despite adjuvant RT, while no difference was observed in WHO-type A-B1. This might be due to selection bias, where patients with more aggressive features were treated with surgery plus RT, as in the current study. Thus, based on these data, the role of adjuvant RT in stage II patients with WHO-type B2-C remains inconclusive. Considering an excellent local control rate (100\% in the current study)

Table 5. Reported recurrence rates in patients with completely resected stage II thymoma according to WHO histology

\begin{tabular}{|c|c|c|c|c|}
\hline \multirow{2}{*}{ Study } & \multirow{2}{*}{ Year } & \multirow{2}{*}{ WHO histology } & \multicolumn{2}{|c|}{ No. of recurrence/Total patients (\%) } \\
\hline & & & Surgery & Surgery+RT \\
\hline \multirow[t]{2}{*}{ Singhal et al. [13] } & \multirow{2}{*}{2003} & A-B1 & $0 / 12(0.0)$ & $0 / 12(0.0)$ \\
\hline & & B2-C & $0 / 8(0.0)$ & $1 / 8(12.5)$ \\
\hline \multirow[t]{2}{*}{ Rena et al. [12] } & \multirow{2}{*}{2007} & A-B1 & $2 / 26(7.7)$ & $2 / 19(10.5)$ \\
\hline & & B2-C & $0 / 7(0.0)$ & $1 / 6(16.6)$ \\
\hline \multirow[t]{2}{*}{ Chen et al. [10] } & \multirow{2}{*}{2010} & A-B1 & $1 / 32(3.0)$ & $2 / 46(4.3)$ \\
\hline & & B2-C & $1 / 7(14.3)$ & $3 / 16(18.7)$ \\
\hline \multirow[t]{2}{*}{ Current study } & \multirow{2}{*}{2011} & A-B1 & $0 / 7(0.0)$ & $0 / 8(0.0)$ \\
\hline & & B2-C & $0 / 2(0.0)$ & $1 / 13(7.7)$ \\
\hline \multirow[t]{2}{*}{ Total } & & A-B1 & $3 / 77(3.9)$ & 4/85 (4.7) \\
\hline & & B2-C & $1 / 24(4.1)$ & $6 / 43(13.9)$ \\
\hline
\end{tabular}

WHO, World Health Organization; RT, radiation therapy. 
and low morbidity (no grade 2 or greater toxicity in the current study) in modern 3-dimensional RT, adjuvant RT for patients with WHO-type B2-C may be considered in the lack of the prospective randomized trial.

The current study has a few limitations: retrospective nature; selection bias between treatment groups; and too few events to evaluate the role of adjuvant RT. Considering the rarity and the rather indolent clinical course of TET, multi-institutional prospective clinical study would be desired.

\section{Conclusion}

Excellent outcomes were achieved in patients with stage I-II TET who underwent complete resection. Considering excellent local control and low morbidity, adjuvant RT may be considered in high risk patients with WHO-type B2-C histology and Masaoka stage II.

\section{Conflicts of Interest}

Conflict of interest relevant to this article was not reported.

\section{References}

1. Berrih-Aknin S, Morel E, Raimond F, Safar D, Gaud C, Binet JP, et al. The role of the thymus in myasthenia gravis: immunohistological and immunological studies in 115 cases. Ann N Y Acad Sci. 1987;505:50-70.

2. Rosai J, Sobin LH. World Health Organization: international histological classification of tumours. Histological typing of tumours of the thymus. New York: Springer; 1999

3. Chen G, Marx A, Chen WH, Yong J, Puppe B, Stroebel P, et al. New WHO histologic classification predicts prognosis of thymic epithelial tumors: a clinicopathologic study of 200 thymoma cases from China. Cancer. 2002;95:420-9.

4. Okumura M, Ohta M, Tateyama H, Nakagawa K, Matsumura A, Maeda H, et al. The World Health Organization histologic classification system reflects the oncologic behavior of thymoma: a clinical study of 273 patients. Cancer. 2002;94:624-32.

5. Lee HS, Kim ST, Lee J, Choi YS, Han JH, Ahn YC, et al. A single institutional experience of thymic epithelial tumours over 11 years: clinical features and outcome and implications for future management. Br J Cancer. 2007;97:22-8.

6. Kang MW, Lee ES, Jo J, Han J, Ahn YC, Kim HK, et al. Stage III thymic epithelial neoplasms are not homogeneous with regard to clinical, pathological, and prognostic features. J Thorac Oncol. 2009;:1561-7.

7. Masaoka A, Monden Y, Nakahara K, Tanioka T. Follow-up study of thymomas with special reference to their clinical stages. Cancer. 1981;48:2485-92.

8. Masaoka A, Yamakawa Y, Niwa H, Fukai I, Saito Y, Tokudome S, et al. Thymectomy and malignancy. Eur J Cardiothorac Surg. 1994;8:251-3.

9. Koga K, Matsuno Y, Noguchi M, Mukai K, Asamura H, Goya T, et al. A review of 79 thymomas: modification of staging system and reappraisal of conventional division into invasive and non-invasive thymoma. Pathol Int. 1994;44:359-67.

10. Chen YD, Feng QF, Lu HZ, Mao YS, Zhou ZM, Ou GF, et al. Role of adjuvant radiotherapy for stage II thymoma after complete tumor resection. Int J Radiat Oncol Biol Phys. 2010;78:1400-6.

11. Detterbeck FC. Evaluation and treatment of stage I and II thymoma. J Thorac Oncol. 2010;5(10 Suppl 4):S318-22.

12. Rena O, Papalia E, Oliaro A, Ruffini E, Filosso P, Novero D, et al. Does adjuvant radiation therapy improve disease-free survival in completely resected Masaoka stage II thymoma? Eur J Cardiothorac Surg. 2007:31:109-13.

13. Singhal S, Shrager JB, Rosenthal DI, LiVolsi VA, Kaiser LR. Comparison of stages I-II thymoma treated by complete resection with or without adjuvant radiation. Ann Thorac Surg 2003:76:1635-41.

14. Utsumi T, Shiono H, Kadota Y, Matsumura A, Maeda H, Ohta M, et al. Postoperative radiation therapy after complete resection of thymoma has little impact on survival. Cancer. 2009; 115:5413-20.

15. Detterbeck FC, Parsons AM. Thymic tumors. Ann Thorac Surg. 2004;77:1860-9.

16. Falkson CB, Bezjak A, Darling G, Gregg R, Malthaner R, Maziak DE, et al. The management of thymoma: a systematic review and practice guideline. J Thorac Oncol. 2009;4:911-9.

17. Zhang H, Lu N, Wang M, Gu X, Zhang D. Postoperative radiotherapy for stage I thymoma: a prospective randomized trial in 29 cases. Chin Med J (Engl). 1999;112:136-8.

18. Regnard JF, Magdeleinat P, Dromer C, Dulmet E, de Montpreville V, Levi JF, etal. Prognostic factors and long-term results after thymoma resection: a series of 307 patients. J Thorac Cardiovasc Surg. 1996;112:376-84

19. Ogawa K, Uno T, Toita T, Onishi H, Yoshida H, Kakinohana Y, etal. Postoperative radiotherapy for patients with completely resected thymoma: a multi-institutional, retrospective review of 103 patients. Cancer. 2002;94:1405-13.

20. Haniuda M, Miyazawa M, Yoshida K, Oguchi M, Sakai F, Izuno I, et al. Is postoperative radiotherapy for thymoma effective? Ann Surg. 1996;224:219-24.

21. Curran WJ Jr, Kornstein MJ, Brooks JJ, Turrisi AT 3rd. Invasive thymoma: the role of mediastinal irradiation following complete or incomplete surgical resection. J Clin Oncol. 1988:6:1722-7.

22. Monden Y, Nakahara K, lioka S, Nanjo S, Ohno K, Fujii Y, et al. Recurrence of thymoma: clinicopathological features, therapy, and prognosis. Ann Thorac Surg. 1985;39:165-9.

23. Cowen D, Richaud P, Mornex F, Bachelot T, Jung GM, Mirabel X, et al. Thymoma: results of a multicentric retrospective series of 149 non-metastatic irradiated patients and review of the literature. FNCLCC trialists. Federation Nationale des Centres de Lutte Contre le Cancer. Radiother Oncol. 1995;34:9-16.

24. Latz D, Schraube P, Oppitz U, Kugler C, Manegold C, Flentje M, et al. Invasive thymoma: treatment with postoperative radiation therapy. Radiology. 1997;204:859-64.

25. Korst RJ, Kansler AL, Christos PJ, Mandal S. Adjuvant radiotherapy for thymic epithelial tumors: a systematic review and meta-analysis. Ann Thorac Surg. 2009;87:1641-7. 